\title{
One- and two-neutron removal from the neutron-rich carbon isotopes
}

\author{
E. C. Simpson and J. A. Tostevin \\ Department of Physics, Faculty of Engineering and Physical Sciences, University of Surrey, Guildford, Surrey GU2 7XH, United Kingdom
}

(Received 10 November 2008; published 25 February 2009)

\begin{abstract}
Reactions that involve the fast removal (knockout) of one and two neutrons from the neutron-rich carbon isotopes, ${ }^{15-19} \mathrm{C}$, by light nuclear targets are studied within an eikonal reaction model. Shell model calculations are used to describe the excitation energy spectra and the structures of the carbon isotopes. The calculated one-neutron knockout cross sections from the ${ }^{A} \mathrm{C}$ isotopes, to particle-bound configurations of the ${ }^{A-1} \mathrm{C}$ residues, are in agreement with the available experimental data. The two-neutron removal cross sections, producing ${ }^{A-2} \mathrm{C}$ residues, receive contributions from both the direct, single-step two-neutron knockout and the indirect mechanism, involving single-neutron removal strength to neutron-unbound excited states in the ${ }^{A-1} \mathrm{C}$ system followed by neutron emission. The latter two-step reaction mechanism is shown to be dominant. The empirical odd-even staggering of the single-neutron separation energies along the carbon isotopic chain is reflected in the two-neutron removal data. This staggering and the magnitudes of the two-neutron removal cross sections are reproduced qualitatively by the theoretical calculations.
\end{abstract}

DOI: 10.1103/PhysRevC.79.024616

PACS number(s): 24.50.+g, 21.10.Jx, 25.60.Gc, 27.20.+n

\section{INTRODUCTION}

One-nucleon knockout reactions from intermediate-energy beams have been extensively studied for projectile nuclei with masses in the range $A \approx 10-60$. These reaction data, which measure only the fast, forward-going mass $A-1$ residues, are thus inclusive with respect to the final state of the target nucleus but can be either inclusive or exclusive with respect to the (bound) final states of the mass $A-1$ residues. The reactions have been used in studies of very asymmetric nuclei produced as fast (but low intensity) secondary radioactive beams, and analyses now include transitions due to the removal of both strongly bound and weakly bound nucleons, see, e.g., Refs. [1-3]. More recently, reaction studies have exploited the direct nature of the reaction mechanism for the removal of two well-bound (like) nucleons from already asymmetric nuclei to populate and probe the structure of the most neutron-rich and neutron-deficient nuclei [4-7]. These analyses exploit the accuracy and simplicity of the eikonal model for the reaction dynamics at the intermediate energies, of order $100 \mathrm{MeV} /$ nucleon, of fragmentation facilities [5,7].

A significant complication arises in the case of the removal of two weakly bound nucleons from a mass $A$ projectile, for example, in two-neutron knockout from neutron-rich isotopes. Here, the mass $A-2$ residues will result not only from the fast single-step direct knockout of a pair of neutrons but also from events in which a neutron is emitted from particle-unbound excited states of the intermediate-mass $A-1$ residues populated by the stronger single-neutron knockout mechanism. This paper presents a first quantitative estimate of the relative strengths of these direct and indirect two-neutron removal mechanisms and their systematics in reactions along the carbon isotopic chain: for ${ }^{15-19} \mathrm{C}$. Such an assessment is essential to understanding the extent to which experiments that measure only the mass $A-2$ residues (but not the removed neutrons) may be sensitive to, and might be able to identify, novel two-neutron correlations in the surface of neutron-rich asymmetric systems, i.e., cases where the excess neutrons are weakly bound.
The aim of the present study is to carry out a systematic first analysis of available measurements of one- and two-neutron knockout data for the neutron-rich carbon isotopes ${ }^{15-19} \mathrm{C}$. Our goals are (i) to compare the predicted and measured oneand two-neutron removal cross sections across all isotopes for which data are available and (ii) to investigate the systematics and relative strengths of the contributions to the two-neutron removal yields from direct (one-step) and indirect (two-step) processes. Goals (i) and (ii) require a consistent structure model for neutrons at the boundary with the $p$ shell and in the lower part of the $s d$ shell, including predictions of the oneand two-neutron strength distributions relative to the neutron thresholds. Only the shell model provides this information readily in a form that can be incorporated into the reaction model. The latter uses a consistent model for the neutron- and residue-target interactions based on reaction cross section and density systematics.

In Sec. II we discuss the knockout model used and our approach to calculating the one- and two-neutron removal cross sections from the neutron-rich isotopes. We then discuss the inputs, namely, the eikonal model elastic $S$ matrices, neutron bound states wave functions, and the shell model spectroscopic strengths, needed for the model calculations of Sec. III. The results of our calculations of inclusive cross sections for both one- and two-neutron removal from ${ }^{15-19} \mathrm{C}$ and for the heavy-fragment parallel momentum distributions for knockout reactions from ${ }^{16} \mathrm{C}$ and ${ }^{19} \mathrm{C}$ projectiles are presented in Sec. IV. The results are summarized in the concluding section.

\section{KNOCKOUT REACTION ANALYSES}

Fast one- and two-neutron removal reactions from the neutron-rich carbon isotopes have been studied experimentally by several authors [8-15] using either beryllium $\left({ }^{9} \mathrm{Be}\right)$ or carbon $\left({ }^{12} \mathrm{C}\right)$ targets. The incident beam energies of these data sets range from 45 to $103 \mathrm{MeV} /$ nucleon. In several cases, parallel momentum distributions of the reaction residues were 


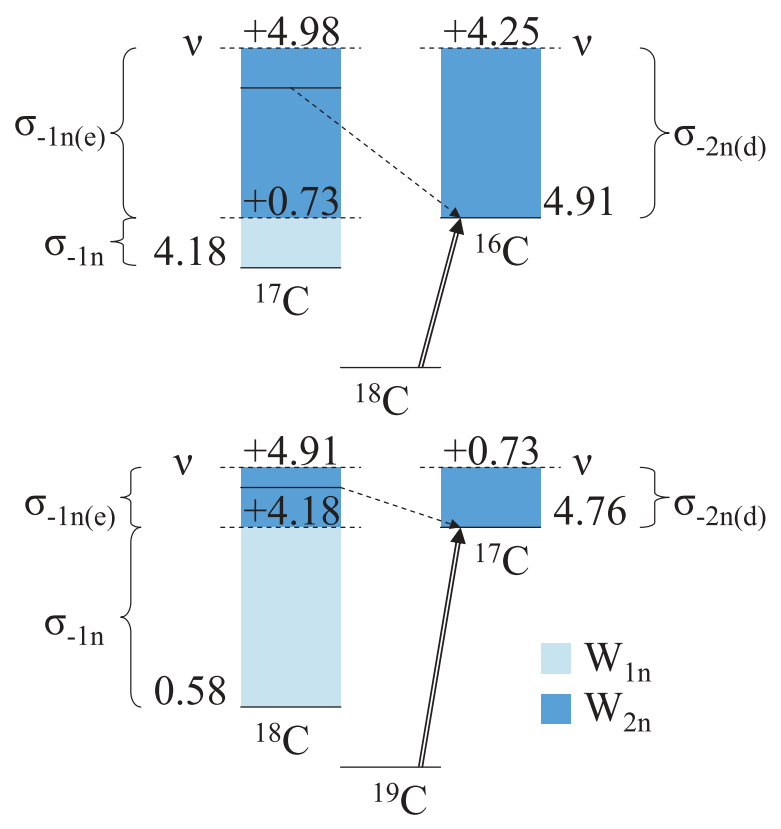

FIG. 1. (Color online) Schematic energy diagram showing the relevant one- and two-neutron thresholds for knockout reactions from neutron-rich ${ }^{18} \mathrm{C}$ (upper part) and from ${ }^{19} \mathrm{C}$ (lower part). The energy intervals for transitions applicable to the inclusive cross sections for the indirect two-neutron removal (one-neutron removal followed by neutron emission), $\sigma_{-1 n(e)}$, and direct two-neutron removal, $\sigma_{-2 n(d)}$, reaction mechanisms are shown. These are seen to be larger for reactions from the even- $A$ isotopes (upper part).

also measured. The experimental two-neutron removal cross sections, $\sigma_{-2 n}$, show a pronounced odd-even mass staggering, the cross section being enhanced significantly for removal from the even- $A$ isotopes. We note that the ground-state to ground-state one-neutron separation energies also exhibit such a staggering with $A$, as is shown in detail for the ${ }^{18} \mathrm{C}$ and ${ }^{19} \mathrm{C}$ cases in Fig. 1. Evident from the figure is that these trends in the separation energies result in relatively large energy windows for two-neutron knockout from beams of even $A$. For example, in the upper part of the figure, for ${ }^{18} \mathrm{C}$, there is an extended (bound final states) energy interval below the first neutron threshold in the $A-2$ system. ${ }^{16} \mathrm{C}$ states in this window, $W_{2 n}$, can be accessed by the direct two-neutron removal mechanism. Similarly, there is an extended energy interval between the first and second neutron thresholds in the $A-1$ system. Thus, the neutron single-particle strength in ${ }^{17} \mathrm{C}$ in this (neutron-unbound) energy interval, accessible via one-neutron removal from ${ }^{18} \mathrm{C}$, will contribute to indirect two-neutron removal yield. The converse is true for the carbon isotopes with odd $A$, as is shown in detail in the lower part of Fig. 1 for ${ }^{19} \mathrm{C}$. The trend across the chain of isotopes studied is shown in Fig. 2.

The theoretical model cross sections for one-nucleon knockout to each final state, of spin-parity $J^{\pi}$, are calculated using

$$
\sigma_{-1 n}=\sum_{n \ell j}\left[\frac{A}{A-1}\right]^{N} C^{2} S\left(J^{\pi}, n \ell j\right) \sigma_{\mathrm{sp}}\left(n \ell j, S_{N}^{\mathrm{eff}}\right)
$$

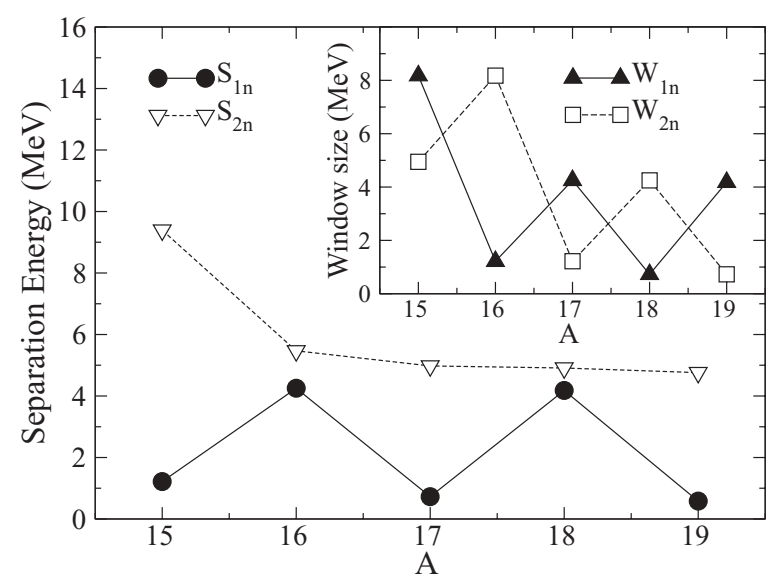

FIG. 2. Ground-state to ground-state one- and two-neutron separation energies for the neutron-rich carbon isotopes (main plot), from Ref. [17]. The inset shows the energy windows for one- and two-neutron knockout transitions, defined as $W_{1 n}=S_{1 n}(A-1)$ and $W_{2 n}=S_{2 n}(A-1)-S_{1 n}(A-1)=S_{1 n}(A-2)$. Population of bound states in ${ }^{17} \mathrm{C}$, in two-neutron removal from ${ }^{19} \mathrm{C}$, requires either (a) direct population of ${ }^{17} \mathrm{C}$ bound states with excitation energy $E^{*}<S_{1 n}(17)$ or (b) population of excited particle-unbound states in ${ }^{18} \mathrm{C}$ with $S_{1 n}(18)<E^{*}<S_{2 n}(18)$. Both of these windows are narrow for the odd- $A$ carbon isotopes, with the expectation of suppressed two-neutron removal cross sections.

where the $C^{2} S$ are the shell model spectroscopic factors and the single-particle cross section $\sigma_{\mathrm{sp}}$ is calculated using the eikonal model assuming unit spectroscopic factor. The quantum numbers of the removed neutron are denoted by $n \ell j$ and $S_{N}^{\mathrm{eff}}$ is the effective separation energy of the neutron for the given final state. The single-particle cross sections $\sigma_{\mathrm{sp}}$ include the contributions from both the stripping (absorption) and diffractive dissociation (elastic breakup) mechanisms. The detailed formulas for the contributions to $\sigma_{\mathrm{sp}}$ from these two mechanisms were presented in Ref. [16]. We assume here, as there, that the heavy residue-target interaction and $S$ matrix are diagonal with respect to the different residue states $J^{\pi}$ and thus that there is no dynamical excitation of the residue in the reaction. The consequence for the present work is that, for the odd- $A$ carbon projectiles where different neutron orbitals $(j)$ can contribute to a given $J^{\pi}$ final state, they do so incoherently. Equation (1) shares this feature with conventional (e.g., distorted-wave Born approximation, DWBA) reaction model expressions for unpolarized transfer reaction cross sections.

In direct two-neutron removal, the theoretical cross sections do not factor into a structure (spectroscopic) factor and a single-particle cross section. The cross section involves the coherent sum over all configurations with nonvanishing shell model two-nucleon amplitudes (TNA), as discussed fully in Refs. [5,7]. For example, the cross section for the stripping of two nucleons populating a particular final state $f$ is given by

$$
\sigma_{\mathrm{str}}^{f}=\frac{1}{2 J_{i}+1} \int d \vec{b} \sum_{M_{i} M_{f}}\left\langle\Psi_{J_{i} M_{i}}^{(f)}|\mathcal{A}| \Psi_{J_{i} M_{i}}^{(f)}\right\rangle
$$


with $\mathcal{A} \equiv \mathcal{A}(\vec{b}, 1,2)$ the stripping mechanism joint probability

$$
\mathcal{A}(\vec{b}, 1,2)=\left|\mathcal{S}_{c}\right|^{2}\left(1-\left|\mathcal{S}_{1}\right|^{2}\right)\left(1-\left|\mathcal{S}_{2}\right|^{2}\right),
$$

and the two-nucleon overlaps are given by [5]

$$
\begin{aligned}
\Psi_{J_{i} M_{i}}^{(f)} & \equiv\left\langle\Phi_{J_{f} M_{f}}(A-2) \mid \Psi_{J_{i} M_{i}}(A)\right\rangle \\
& =\sum_{I \mu \alpha} C_{\alpha}^{J_{i} J_{f} I}\left(I \mu J_{f} M_{f} \mid J_{i} M_{i}\right)\left[\overline{\phi_{j_{1}} \otimes \phi_{j_{2}}}\right]_{I \mu} .
\end{aligned}
$$

Here, $\Psi_{J_{i} M_{i}}(A)$ is the $A$-body projectile wave function, and $\Phi_{J_{f} M_{f}}(A-2)$ is the $A-2$ body residue (core) wave function, in one of a number of final states $f$. The normalized removednucleon single-particle wave functions are $\phi_{j}$. The TNA are the coefficients $C_{\alpha}^{J_{i} J_{f} I}$, given by shell model calculations, and contain the structure details, in particular, the parentage and phase of each contributing two-nucleon configuration. As was discussed for the one-neutron removal case, in writing Eq. (2) we made use of the spectator-core approximation with the result that for the odd- $A$ carbon projectiles, where more than one two-neutron pair total angular momentum $I$ is possible, these contribute incoherently to each $J^{\pi}$ final state. This was shown explicitly in Ref. [5]. While not pursued further here, we note that the residue parallel momentum distributions following direct two-nucleon removal reactions depend strongly on the total angular momentum $I$ of the pair of removed nucleons $[18,19]$. Further, these analyses show that the spatial and impact-parameter selectivity and sensitivity of the stripping and diffraction mechanisms are very similar.

Here, we include full calculations of the direct two-neutron removal yields from both (i) two-neutron stripping, the $\sigma_{\text {str }}$ of Eq. (2), and (ii) one-neutron being stripped (labeled $i=1,2$ ) and the other diffracted, denoted $\sigma_{\text {diff, } i}$ [7]. Thus, because of the two removed neutrons, this total stripping-diffraction cross section is $\sigma_{\mathrm{ds}}=\sigma_{\mathrm{diff}, 1}+\sigma_{\mathrm{diff}, 2}$.

The two-neutron diffraction events are only estimated. Such an estimate can be made in two ways. The first (preferred) method, following Ref. [7], makes use of the ratio of the cross sections obtained when one neutron is stripped and the other elastically dissociated, $\sigma_{\text {diff }, i}$, compared with both neutrons being stripped, $\sigma_{\text {str }}$, as obtained above. That is, assuming all removal mechanisms have similar spatial and impact-parameter selectivity, e.g., Refs. [18,19], we estimate the probability of two-neutron diffraction events relative to two-neutron stripping events as $\sigma_{\mathrm{diff}} / \sigma_{\mathrm{str}} \approx\left[\sigma_{\mathrm{diff}, i} / \sigma_{\mathrm{str}}\right]^{2}$. This is our preferred estimate, since the calculations of both $\sigma_{\text {diff }, i}$ and $\sigma_{\text {str }}$ include all spatial correlations present in the shell model two-neutron overlaps. For the cases considered here, we typically find that $\sigma_{\mathrm{diff}, i} / \sigma_{\mathrm{str}} \approx 0.40-.45$ and hence that $\sigma_{\text {diff }}$ makes a contribution of about $6-9 \%$ of the total direct two-neutron removal cross sections.

A second estimate of the importance of $\sigma_{\text {diff }}$ can be obtained if we take instead our estimate of the probability of neutron diffraction to stripping events from the diffractive and stripping contributions of the one-neutron removal cross sections $\sigma_{\mathrm{sp}}$, but calculated using the average separation energy of the two removed neutrons. This simpler estimate clearly does not take account of two-neutron correlations present in the shell model wave functions. Nevertheless, it provides a similar estimate of the predicted $\sigma_{\text {diff }} / \sigma_{\text {str }}$ ratio, giving $11 \%$ of the total direct two-neutron removal cross sections in the ${ }^{15} \mathrm{C}$ projectile case.

We will see in the following that indirect two-neutron removal, involving single-neutron removal strength to neutronunbound excited states in the ${ }^{A-1} \mathrm{C}$ system followed by neutron emission, dominates over the direct two-neutron removal mechanisms above. Thus, while the calculation of $\sigma_{\text {diff }}$ should be improved in subsequent fully quantitative studies, and will be needed as and when experiments are able to disentangle the direct two-neutron removal contribution, the estimate above is sufficient for the inclusive and still rather low precision data available to date.

\section{MODEL INPUTS}

We adopt the eikonal model description of the reaction mechanism. Given an interaction description, the eikonal approximation has been shown [20] to provide a rather accurate description of the elastic $S$ matrix and derived observables for incident projectile energies of $50 \mathrm{MeV} /$ nucleon and greater. There are also strategies known to improve the description of the $S$ matrix to lower beam energies if required, e.g., in Refs. [21,22]. The underlying model assumptions improve as the beam energies increase, so, in the following, when multiple data sets are available for a given projectile, we perform calculations for the highest incident beam energy for which data are available. In the following, only one data set is at $64 \mathrm{MeV} /$ nucleon. All other data are at $80 \mathrm{MeV} /$ nucleon or higher.

More details of our eikonal model implementation can be found in Refs. [2,3,7]. The required neutron- and residue-target elastic $S$ matrices were calculated using the static density limit of the eikonal model [23], or equivalently, the singleand double-folding models, respectively, using the residue and target point neutron and proton densities and an effective nucleon-nucleon $(N N)$ interaction. The densities for the mass $A-1$ and $A-2$ residues from each projectile were taken from spherical Skyrme (SkX) Hartree-Fock (HF) calculations [24]. The densities of the carbon (beryllium) target nuclei were assumed to be of Gaussian form with a rms matter radius of 2.32 (2.36) fm. A finite-range, Gaussian $N N$ effective interaction of range parameter $0.5 \mathrm{fm}$ was used. Its strength was calculated from the free neutron-neutron and neutron-proton cross sections appropriate to the beam energy and from the real-to-imaginary ratios of the $N N$ forward scattering amplitudes at the lowest energy tabulated by Ray [25], this being for a beam energy of $100 \mathrm{MeV} /$ nucleon.

This parameter choice generates neutron-target $S$ matrices that reproduce the measured neutron-target reaction cross sections and have also been shown, e.g., in Ref. [26], to reproduce calculations of exclusive neutron-removal cross sections based instead upon the microscopic $G$ matrix interaction of Jeukenne, Lejeune, and Mahaux [27]. Most recently, this parameter choice has also been shown to reproduce the ratios of the diffraction and stripping mechanism yields, now measured precisely in ${ }^{8} \mathrm{~B}$ and ${ }^{9} \mathrm{C}$ induced reactions by Bazin et al. [28], and confirming an earlier but poorer statistics analysis by Enders et al. [29]. 
Were we to extrapolate (by polynomial fit) the parameters of Ray [25] below their tabulated range, i.e., $<100 \mathrm{MeV} /$ nucleon, we would obtain a small enhancement of the diffractive cross section, of $\approx 10 \%$ at $80 \mathrm{MeV} / \mathrm{u}$ and $\approx 15 \%$ at $60 \mathrm{MeV} / \mathrm{u}$. Of course all stripping cross sections, whose dependence on the $S$ matrices are through $|\mathcal{S}|^{2}$, remain unchanged. This small, $<100 \mathrm{MeV}$ beam energy uncertainty has no bearing on the conclusions that we draw here.

Whereas in one-nucleon knockout studies that attempt to extract absolute spectroscopic strengths from experimental data, see, e.g., Ref. [3], the removed nucleon's wave functions are also constrained using HF inputs, in the present work the wave functions of all bound states are calculated in a Woods-Saxon well of a fixed geometry. All neutron singleparticle radial wave functions were calculated using a standard Woods-Saxon potential geometry with a radius parameter $r_{0}=1.25 \mathrm{fm}$ and a diffuseness $a_{0}=0.7 \mathrm{fm}$. The sensitivity to this choice has been shown to be small [3]. The depths of the potential wells in each case were adjusted to reproduce the empirical separation energy, and the excitation energy of each final state was taken into account. The ground-state to ground-state one- and two-neutron separation energies were taken from the 2003 mass compilation [17].

Shell model calculations were used for the required level energies, spectroscopic factors, and two-nucleon amplitudes. These were performed using the code OXBASH [30]. The calculations used the WBP effective interaction [31], and a model space truncated to allow $0 \hbar \omega$ and $1 \hbar \omega$ excitations relative to the $p$-sd ground state. The small center-of-mass correction factor $[A /(A-1)]^{N}$, shown in Eq. (1), with $N$ the principal oscillator model quantum number of the removed-nucleon shell [32], was applied to the shell model spectroscopic factors in all single-neutron removal calculations. We note, however, that in its formulation, it is assumed that the removed neutron occupies the highest occupied oscillator shell in the initial state. No such corrections were applied to the two-nucleon amplitudes.

The theoretical inclusive one- and two-neutron removal cross sections will clearly be sensitive to which excited states the shell model calculations predict to lie above or below the relevant neutron thresholds. A general tendency of oscillatorbased shell model calculations is for theoretical excited state energies to overestimate those measured. Thus, there is some degree of ambiguity as to how much of the single-particle strength the shell model places in a given physical excitation energy window. In the following, the one-neutron removal cross sections were calculated for all final states of significant spectroscopic strength in the relevant energy windows. To estimate the sensitivity to these spectroscopy details, in the case of the indirect two-neutron removal mechanism, we have also calculated one-neutron removal cross sections for states up to $1.5 \mathrm{MeV}$ above the two-neutron thresholds. In so doing, we quantify possible additional contributions arising from states predicted by the shell model to be two-neutron unbound, but which may in reality be unbound only to one-particle emission.

In essentially all cases, the one- and two-particle strengths predicted (by the shell model) to lie below the physical neutron thresholds are consistent with the empirical reaction yields. However, for ${ }^{16} \mathrm{C}$ and ${ }^{18} \mathrm{C}$, states predicted by the shell-model to lie above the neutron thresholds have been observed experimentally as particle-bound states. Generally, the experimentally observed levels are lower in energy than those predicted theoretically. Thus, in the following, when experimental energies are available for strong single-particle transitions, these empirical energies have been used. These cases are identified in the tables of results in Sec. IV. These reduced final state excitation energies clearly reduce the effective neutron separation energies $S_{n}^{\text {eff }}$ entering the reaction calculations resulting in a modest enhancement of the theoretical single-particle cross sections to these excited states.

In ${ }^{16} \mathrm{C}$, the $2^{+}, 3^{+}$, and $4^{+}$states are seen experimentally near $4.1 \mathrm{MeV}$, and below the one-neutron threshold of 4.18 MeV. As in the work of Maddalena et al. [9], we have identified these states with the corresponding shell model states at 4.7-5.7 MeV and placed them as a group at 4.1 MeV. In the case of ${ }^{19} \mathrm{C} \rightarrow{ }^{18} \mathrm{C}$, the WBP shell model calculations place several states between the one- and two-neutron threshold energies of ${ }^{18} \mathrm{C}$, specifically the $J^{\pi}=1^{-}$state at $4.437 \mathrm{MeV}$ and the $2^{+}$and $3^{+}$states at 4.915 and $4.975 \mathrm{MeV}$. Maddalena et al. [9] assume that the two states near $4.9 \mathrm{MeV}$ are neutron bound. Calculations using a modified version of the WBT interaction [33] also suggest that the $2^{+}$and $3^{+}$states are bound with respect to neutron emission. The $2^{+}$and $3^{+}$shell model states are thus expected to be bound. In the absence of experimental information for the energy of the $1^{-}$state, we assume the shell model value, and thus the state will contribute to the two-neutron removal cross section.

\section{RESULTS}

We now present the results of the calculations of the inclusive cross sections following one- and two-neutron removal from the ${ }^{15-19} \mathrm{C}$ isotopes. The parallel momentum distributions of the residues following one- and two-neutron removal, from the ${ }^{16} \mathrm{C}$ and ${ }^{19} \mathrm{C}$ systems, are also presented.

\section{A. Results for one-neutron knockout}

The results for the calculated inclusive (and also their component exclusive) one-neutron removal cross sections are presented in Table I. For each isotope $A$, the table shows those neutron states used, together with their associated singleparticle cross sections and spectroscopic factors, to compute each one-neutron removal inclusive cross section $\sigma_{-1 n}$. The calculations are seen to be in reasonable agreement with the experimental values, given their stated errors, and with the previous theoretical analysis of Refs. [9] and [12]. For ${ }^{19} \mathrm{C}$, the evaluated one-neutron separation energy, $S_{1 n}(19)=$ $0.58 \mathrm{MeV}$ [17], is now smaller than that used in Ref. [9], resulting in an enhanced cross section for the $\ell=0$ knockout to the ${ }^{18} \mathrm{C}$ ground state. Unless stated otherwise, see, e.g., the previous section regarding states in ${ }^{16} \mathrm{C}$ and ${ }^{18} \mathrm{C}$, all of the predicted shell model states with excitation energies $E^{*} \leqslant S_{1 n}(A-1)$ are included.

The trends of the cross sections across the full isotopic chain are shown in Fig. 3. The solid line connects the theoretical 
TABLE I. Results for one-neutron knockout reactions to bound final states of ${ }^{14-18} \mathrm{C}$. The calculations are for a ${ }^{12} \mathrm{C}$ target for the ${ }^{16} \mathrm{C},{ }^{17} \mathrm{C}$, and ${ }^{18} \mathrm{C}$ projectiles at 83,79 , and $80 \mathrm{MeV} /$ nucleon, respectively, and for a ${ }^{9} \mathrm{Be}$ target for ${ }^{15} \mathrm{C}$ and ${ }^{19} \mathrm{C}$ at 103 and $64 \mathrm{MeV} /$ nucleon. All cross sections are in $\mathrm{mb}$. The cross sections $\sigma_{-1 n}$ include the center-of-mass correction factor $[A /(A-1)]^{N}$ [32]. The effective final state excitation energy $\bar{E}_{f}$ used to compute the average Fermi-surface asymmetry for the removed neutrons, $\Delta S^{\text {eff }}=\left[S_{1 n}+\bar{E}_{f}\right]-S_{1 p}$, was obtained by weighting the excitation energy of each shell model state by the calculated neutron removal cross section to that state. The errors shown for $R_{s}$ are based only upon the errors quoted on the experimental measurements.

\begin{tabular}{|c|c|c|c|c|c|c|c|c|c|}
\hline Reaction & $E_{x}(\mathrm{MeV})$ & $J^{\pi}$ & $\ell$ & $\sigma_{\text {str }}(\mathrm{mb})$ & $\sigma_{\text {dif }}(\mathrm{mb})$ & $C^{2} S$ & $\sigma_{-1 n}(\mathrm{mb})$ & $\sigma_{\exp }(\mathrm{mb})$ & $R_{s}$ \\
\hline$\left({ }^{15} \mathrm{C},{ }^{14} \mathrm{C}\right)[12]$ & 0.000 & $0^{+}$ & 0 & 70.9 & 38.9 & 0.978 & 123.3 & $100.8(44)$ & $0.82^{\mathrm{a}}$ \\
\hline$\Delta S^{\mathrm{eff}}=-18.0 \mathrm{MeV}$ & $6.094^{\mathrm{b}}$ & $1^{-}$ & 1 & 22.4 & 6.1 & 1.180 & 36.0 & $27.4(41)$ & 0.76 \\
\hline \multirow[t]{3}{*}{$S_{1 n}(14)=8.176$} & $6.903^{\mathrm{b}}$ & $0^{-}$ & 1 & 21.4 & 5.6 & 0.459 & 13.3 & $6.5(9)$ & 0.49 \\
\hline & $7.012^{\mathrm{b}}$ & $2^{+}$ & 2 & 22.9 & 5.7 & 0.020 & 0.7 & $5.5(17)$ & - \\
\hline & & Inclusive & & & & & 173.3 & $140.2 \pm 4.6$ & $0.81 \pm 0.03$ \\
\hline$\left({ }^{16} \mathrm{C},{ }^{15} \mathrm{C}\right)[10]$ & 0.000 & $1 / 2^{+}$ & 0 & 42.4 & 19.7 & 0.601 & 42.5 & & \\
\hline$\Delta S^{\mathrm{eff}}=-17.9 \mathrm{MeV}$ & $0.740^{\mathrm{b}}$ & $5 / 2^{+}$ & 2 & 25.3 & 8.7 & 1.232 & 47.8 & & \\
\hline$S_{1 n}(15)=1.218$ & & Inclusive & & & & & 90.2 & $65_{-10}^{+15}$ & $0.72_{-0.11}^{+0.17}$ \\
\hline$\left({ }^{17} \mathrm{C},{ }^{16} \mathrm{C}\right)[14]$ & 0.000 & $0^{+}$ & 2 & 38.9 & 18.8 & 0.035 & 2.3 & & \\
\hline$\Delta S^{\mathrm{eff}}=-20.1 \mathrm{MeV}$ & $1.766^{\mathrm{b}}$ & $2^{+}$ & 0 & 53.3 & 28.4 & 0.163 & 15.0 & & \\
\hline \multirow[t]{4}{*}{$S_{1 n}(16)=4.250$} & & & 2 & 29.6 & 12.0 & 1.445 & 67.9 & & \\
\hline & $4.100^{c}$ & $2^{+}, 3^{(+)}, 4^{+}$ & 0 & 37.5 & 17.1 & 0.225 & 13.9 & & \\
\hline & & & 2 & 24.0 & 8.5 & 0.770 & 28.3 & & \\
\hline & & Inclusive & & & & & 127.3 & $116 \pm 18$ & $0.93 \pm 0.14$ \\
\hline$\left({ }^{18} \mathrm{C},{ }^{17} \mathrm{C}\right)[15]$ & 0.000 & $3 / 2^{+}$ & 2 & 23.6 & 8.7 & 0.103 & 3.7 & & \\
\hline$\Delta S^{\mathrm{eff}}=-21.8 \mathrm{MeV}$ & 0.032 & $5 / 2^{+}$ & 2 & 23.6 & 8.7 & 2.800 & 101.4 & & \\
\hline \multirow[t]{2}{*}{$S_{1 n}(17)=0.727$} & 0.295 & $1 / 2^{+}$ & 0 & 36.9 & 17.1 & 0.654 & 39.6 & & \\
\hline & & Inclusive & & & & & 144.7 & $155 \pm 24$ & $1.07 \pm 0.17$ \\
\hline$\left({ }^{19} \mathrm{C},{ }^{18} \mathrm{C}\right)[13]$ & 0.000 & $0^{+}$ & 0 & 95.0 & 65.6 & 0.580 & 103.7 & & \\
\hline$\Delta S^{\mathrm{eff}}=-23.5 \mathrm{MeV}$ & 2.144 & $2^{+}$ & 2 & 26.0 & 10.3 & 0.470 & 19.0 & & \\
\hline \multirow[t]{5}{*}{$S_{1 n}(18)=4.180$} & 3.639 & $2^{+}$ & 2 & 22.4 & 8.1 & 0.104 & 3.5 & & \\
\hline & 3.988 & $0^{+}$ & 0 & 34.5 & 15.6 & 0.319 & 17.8 & & \\
\hline & 4.915 & $3^{+}$ & 2 & 20.3 & 6.9 & 1.523 & 46.2 & & \\
\hline & 4.975 & $2^{+}$ & 2 & 20.3 & 6.8 & 0.922 & 27.8 & & \\
\hline & & Inclusive & & & & & 218.0 & $226 \pm 65$ & $1.04 \pm 0.30$ \\
\hline
\end{tabular}

$\overline{\bar{a} \text { Terry } \text { et al. deduce the value } R_{s}=0.90(4)(5) \text { from an analysis of high precision, exclusive data for the } 1 s_{1 / 2} \text { ground-state to ground-state }}$ transition from ${ }^{15} \mathrm{C}$.

${ }^{\mathrm{b}}$ Experimental energy.

${ }^{\mathrm{c}}$ In the ${ }^{16} \mathrm{C}$ projectile case, several predicted shell model states near the two-neutron threshold have been combined and calculated at the threshold energy.

calculations of $\sigma_{-1 n}$ for the reactions of Table I, without any scaling. All available data sets, shown by the symbols (see the figure caption), are well described. The experimental data follow the trends suggested by the size of the energy windows available for the population bound final states. This simple pattern is however distorted as a result of the enhancement of cross sections due to the weaker neutron binding in the odd $A$ projectiles. In the case of ${ }^{18} \mathrm{C}$, the cross section is enhanced over what might be expected from the simple energy window argument due to the existence of the two low-lying excited states below the (small) one-neutron separation energy in ${ }^{17} \mathrm{C}$, resulting in a large spectroscopic strength in the narrow available energy window.

While not a primary object of the present study, the suppression factors $R_{S}=\sigma_{\text {exp }} / \sigma_{-1 n}$ deduced from the experimental and theoretical inclusive cross sections are also shown in Table I. While the error bars (computed from the experimental error) are significant, these $R_{s}$ are near unity and are consistent with the $R_{s}$ systematics computed for a wide range of systems, asymmetries and separation energies, shown most recently in Figure 6 of reference [3]. The effective differences in the energies of the neutron and proton Fermi surfaces, $\Delta S^{\text {eff }}(A)=$ $\left[S_{1 n}(A)+\bar{E}_{f}(A-1)\right]-S_{1 p}(A)$, obtained by weighting the excitation energy $E^{*}$ of each shell model final state by the calculated neutron removal cross section to that state, are also given in the Table.

\section{B. Results for two-neutron knockout}

Table II shows the cross sections due to indirect two-neutron removal, $\sigma_{-1 n(e)}$, resulting from single-neutron knockout to unbound states in the $A-1$ daughter. The single-particle cross sections and shell model spectroscopic factors used are also collected there. We include all states of significant spectroscopic strength below the two-neutron separation energy $S_{2 n}(A-1)$ of the $A-1$ daughter. As was discussed 
TABLE II. Calculated cross sections for one-neutron knockout reactions from ${ }^{15-19} \mathrm{C}$ to unbound states of the intermediate mass $A-1$ nuclei ${ }^{14-18} \mathrm{C}$. The calculations are for a ${ }^{12} \mathrm{C}$ target for the ${ }^{15} \mathrm{C},{ }^{16} \mathrm{C},{ }^{17} \mathrm{C}$, and ${ }^{18} \mathrm{C}$ projectiles at $83,83,79$, and $80 \mathrm{MeV} /$ nucleon, respectively, and for a ${ }^{9} \mathrm{Be}$ target for ${ }^{19} \mathrm{C}$ at $64 \mathrm{MeV} /$ nucleon. The cross sections, $\sigma_{-1 n(e)}$, are calculated on the energy intervals $\left[S_{1 n} \rightarrow S_{2 n}\right.$, where $S_{1 n}$ and $S_{2 n}$ are the one- and two-neutron thresholds of the intermediate mass $A-1$ nucleus. The $\sigma_{-1 n(e)}$ are assumed to contribute to the two-neutron removal reactions $\left({ }^{A} \mathrm{C},{ }^{A-2} \mathrm{C}\right)$ shown, following neutron emission, and include the c.m. correction factor $[A /(A-1)]^{N}$.

\begin{tabular}{|c|c|c|c|c|c|c|c|}
\hline Reaction & $E_{x}(\mathrm{MeV})$ & $J^{\pi}$ & $\ell$ & $\sigma_{\text {str }}(\mathrm{mb})$ & $\sigma_{\mathrm{dif}}(\mathrm{mb})$ & $C^{2} S$ & $\sigma_{-1 n(e)}(\mathrm{mb})$ \\
\hline$\left({ }^{15} \mathrm{C},{ }^{14} \mathrm{C}\left(J^{\pi}\right) \rightarrow{ }^{13} \mathrm{C}\right)[34]$ & 10.219 & $1^{-}$ & 1 & 18.1 & 5.1 & 0.557 & 13.8 \\
\hline \multirow{6}{*}{$E_{x} \in[8.176 \rightarrow 13.122] \mathrm{MeV}$} & 10.259 & $2^{-}$ & 1 & 18.0 & 5.1 & 0.213 & 5.3 \\
\hline & 11.168 & $2^{-}$ & 1 & 17.3 & 4.8 & 0.505 & 12.0 \\
\hline & 12.744 & $2^{-}$ & 1 & 16.3 & 4.3 & 0.418 & 9.3 \\
\hline & & Inclusive & & & & & 40.4 \\
\hline & 13.230 & $1^{-}$ & 1 & 16.0 & 4.2 & 0.394 & 8.6 \\
\hline & & Inclusive & & & & & 49.0 \\
\hline$\left({ }^{16} \mathrm{C},{ }^{15} \mathrm{C}\left(J^{\pi}\right) \rightarrow{ }^{14} \mathrm{C}\right)[10]$ & 1.518 & $1 / 2^{-}$ & 1 & 23.4 & 8.4 & 1.650 & 56.0 \\
\hline \multirow{8}{*}{$E_{x} \in[1.218 \rightarrow 9.394] \mathrm{MeV}$} & 3.804 & $3 / 2^{-}$ & 1 & 19.8 & 6.4 & 0.421 & 11.7 \\
\hline & 4.513 & $3 / 2^{+}$ & 2 & 20.7 & 6.2 & 0.148 & 4.5 \\
\hline & 5.058 & $3 / 2^{-}$ & 1 & 18.4 & 5.7 & 0.674 & 17.3 \\
\hline & 5.925 & $3 / 2^{-}$ & 1 & 17.5 & 5.2 & 0.254 & 6.2 \\
\hline & 7.099 & $3 / 2^{-}$ & 1 & 16.6 & 4.8 & 0.585 & 13.3 \\
\hline & & Inclusive & & & & & 109.0 \\
\hline & 9.794 & $3 / 2^{-}$ & 1 & 14.8 & 4.0 & 0.336 & 6.7 \\
\hline & & Inclusive & & & & & 115.7 \\
\hline$\left({ }^{17} \mathrm{C},{ }^{16} \mathrm{C}\left(J^{\pi}\right) \rightarrow{ }^{15} \mathrm{C}\right)[14]$ & 5.257 & $1^{-}$ & 1 & 21.4 & 7.8 & 0.032 & 1.0 \\
\hline \multirow{5}{*}{$E_{x} \in[4.250 \rightarrow 5.468] \mathrm{MeV}$} & 5.270 & $2^{-}$ & 1 & 21.4 & 7.7 & 0.801 & 24.8 \\
\hline & & Inclusive & & & & & 25.8 \\
\hline & 6.398 & $1^{-}$ & 1 & 20.8 & 6.8 & 0.416 & 12.2 \\
\hline & 6.514 & $2^{-}$ & 1 & 19.4 & 6.6 & 0.122 & 3.4 \\
\hline & & Inclusive & & & & & 41.4 \\
\hline$\left({ }^{18} \mathrm{C},{ }^{17} \mathrm{C}\left(J^{\pi}\right) \rightarrow{ }^{16} \mathrm{C}\right)[35]$ & 0.783 & $1 / 2^{-}$ & 1 & 22.1 & 8.5 & 1.495 & 48.4 \\
\hline \multirow{9}{*}{$E_{x} \in[0.727 \rightarrow 4.977] \mathrm{MeV}$} & 2.492 & $3 / 2^{-}$ & 1 & 18.8 & 6.6 & 0.411 & 11.1 \\
\hline & 2.895 & $3 / 2^{+}$ & 2 & 19.4 & 6.4 & 0.134 & 3.9 \\
\hline & 3.567 & $3 / 2^{-}$ & 1 & 17.3 & 5.8 & 0.397 & 9.7 \\
\hline & 4.515 & $3 / 2^{+}$ & 2 & 17.9 & 5.6 & 0.091 & 2.4 \\
\hline & 4.966 & $3 / 2^{-}$ & 1 & 15.8 & 5.1 & 0.122 & 2.7 \\
\hline & & Inclusive & & & & & 78.2 \\
\hline & 5.876 & $3 / 2^{-}$ & 1 & 15.0 & 4.7 & 0.202 & 4.2 \\
\hline & 6.040 & $3 / 2^{-}$ & 1 & 14.9 & 4.6 & 0.267 & 5.5 \\
\hline & & Inclusive & & & & & 87.9 \\
\hline$\left({ }^{19} \mathrm{C},{ }^{18} \mathrm{C}\left(J^{\pi}\right) \rightarrow{ }^{17} \mathrm{C}\right)[13]$ & 4.437 & $1^{-}$ & 1 & 20.8 & 7.8 & 0.792 & 23.9 \\
\hline \multirow[t]{3}{*}{$E_{x} \in[4.180 \rightarrow 4.910] \mathrm{MeV}$} & 5.763 & $1^{-}$ & 1 & 18.3 & 6.4 & 0.154 & 4.0 \\
\hline & 6.002 & $0^{-}$ & 1 & 17.9 & 6.2 & 0.384 & 9.8 \\
\hline & & Inclusive & & & & & 37.7 \\
\hline
\end{tabular}

earlier, and to assess the sensitivity of the cross sections to the shell model spectroscopy, we have also calculated cross sections when including the additional contributions from shell model states predicted to lie above but within $1.5 \mathrm{MeV}$ of the two-neutron threshold in the mass $A-1$ system. The calculated direct two-neutron removal cross sections are shown in Table III. For each projectile $A$, we show the contributions where both nucleons are absorbed $\sigma_{\text {str }}$, where one nucleon is absorbed and the other removed via diffraction $\sigma_{\mathrm{ds}}$, and our estimate of events where both neutrons are removed by diffraction $\sigma_{\text {diff. }}$. These cross sections are presented in Fig. 4.
Inspection of the results for the two-neutron knockout cross sections in Tables II and III show the reaction yields to be dominated by the one-neutron knockout plus evaporation process. The measured total two-neutron removal cross sections are generally underestimated, particularly when we might expect an additional modest reduction in the theoretical values due to the suppression of shell model strength. On the other hand, a more general reduction of the shell model state energies would (a) bring more states below the two-neutron thresholds and (b) enhance the cross sections for those states already within the energy window, due to a reduction of separation energies, and would enhance the theoretical values. These possibilities have 
TABLE III. Results of full calculations of the stripping (str) and diffraction/stripping (ds), and the estimated diffraction (diff) contributions to the exclusive direct two-neutron removal cross sections from the neutron-rich carbon isotopes. The calculations are for a ${ }^{12} \mathrm{C}$ target for the ${ }^{15} \mathrm{C},{ }^{16} \mathrm{C},{ }^{17} \mathrm{C}$, and ${ }^{18} \mathrm{C}$ projectiles at $83,83,79$, and $80 \mathrm{MeV} /$ nucleon, respectively, and for a ${ }^{9} \mathrm{Be}$ target for ${ }^{19} \mathrm{C}$ at $64 \mathrm{MeV} /$ nucleon. Their totals for each final state, $\sigma_{-2 n(d)}$, and the inclusive direct two-neutron removal cross sections are also shown.

\begin{tabular}{|c|c|c|c|c|c|c|c|}
\hline Reaction & $E_{x}(\mathrm{MeV})$ & $J^{\pi}$ & $I$ & $\sigma_{\mathrm{str}}(\mathrm{mb})$ & $\sigma_{\mathrm{ds}}(\mathrm{mb})$ & $\sigma_{\text {diff }}(\mathrm{mb})$ & $\sigma_{-2 n(d)}(\mathrm{mb})$ \\
\hline \multirow{8}{*}{$\begin{array}{l}\left({ }^{15} \mathrm{C},{ }^{13} \mathrm{C}\right)[12] \\
S_{1 n}(13)=4.946\end{array}$} & \multirow[t]{2}{*}{0.000} & \multirow[t]{2}{*}{$1 / 2^{-}$} & 0 & 0.44 & 0.33 & 0.06 & 0.83 \\
\hline & & & 1 & 2.78 & 2.20 & 0.44 & 5.42 \\
\hline & \multirow[t]{2}{*}{$3.089^{\mathrm{a}}$} & \multirow[t]{2}{*}{$1 / 2^{+}$} & 0 & 1.40 & 0.86 & 0.13 & 2.39 \\
\hline & & & 1 & 0.46 & 0.26 & 0.04 & 0.76 \\
\hline & \multirow[t]{2}{*}{$3.684^{\mathrm{a}}$} & \multirow[t]{2}{*}{$3 / 2^{-}$} & 1 & 1.68 & 1.19 & 0.21 & 3.08 \\
\hline & & & 2 & 1.22 & 0.79 & 0.13 & 2.14 \\
\hline & \multirow[t]{2}{*}{$3.853^{\mathrm{a}}$} & $5 / 2^{+}$ & 2 & 0.02 & 0.01 & 0.00 & 0.03 \\
\hline & & \multicolumn{2}{|l|}{ Inclusive } & & & & 14.65 \\
\hline$\left({ }^{16} \mathrm{C},{ }^{14} \mathrm{C}\right)[10]$ & 0.000 & $0^{+}$ & 0 & 3.47 & 3.06 & 0.67 & 7.20 \\
\hline \multirow[t]{6}{*}{$S_{1 n}(14)=8.176$} & $6.094^{\mathrm{a}}$ & $1^{-}$ & 1 & 1.04 & 0.74 & 0.13 & 1.91 \\
\hline & $6.728^{\mathrm{a}}$ & $3^{-}$ & 3 & 1.79 & 1.19 & 0.20 & 3.18 \\
\hline & $6.903^{\mathrm{a}}$ & $0^{-}$ & 0 & 0.19 & 0.12 & 0.02 & 0.33 \\
\hline & $7.012^{\mathrm{a}}$ & $2^{+}$ & 2 & 0.15 & 0.12 & 0.02 & 0.29 \\
\hline & \multirow{2}{*}{$7.341^{\mathrm{a}}$} & $2^{-}$ & 2 & 0.66 & 0.41 & 0.06 & 1.13 \\
\hline & & \multicolumn{3}{|l|}{ Inclusive } & & & 14.04 \\
\hline \multirow{7}{*}{$\begin{array}{l}\left({ }^{17} \mathrm{C},{ }^{15} \mathrm{C}\right)[14] \\
S_{1 n}(15)=1.218\end{array}$} & \multirow[t]{2}{*}{0.000} & \multirow[t]{2}{*}{$1 / 2^{+}$} & 1 & 0.06 & 0.05 & 0.01 & 0.12 \\
\hline & & & 2 & 0.78 & 0.64 & 0.13 & 1.55 \\
\hline & \multirow[t]{5}{*}{$0.740^{\mathrm{a}}$} & \multirow[t]{4}{*}{$5 / 2^{+}$} & 1 & 0.02 & 0.01 & 0.00 & 0.03 \\
\hline & & & 2 & 3.53 & 3.02 & 0.65 & 7.19 \\
\hline & & & 3 & 0.38 & 0.32 & 0.07 & 0.77 \\
\hline & & & 4 & 0.51 & 0.41 & 0.08 & 1.00 \\
\hline & & \multicolumn{3}{|l|}{ Inclusive } & & & 10.66 \\
\hline$\left({ }^{18} \mathrm{C},{ }^{16} \mathrm{C}\right)[35]$ & 0.000 & $0^{+}$ & 0 & 3.92 & 3.50 & 0.78 & 8.19 \\
\hline \multirow[t]{6}{*}{$S_{1 n}(16)=4.250$} & $1.766^{\mathrm{a}}$ & $2^{+}$ & 2 & 1.04 & 0.87 & 0.18 & 2.10 \\
\hline & $3.027^{\mathrm{a}}$ & $0^{+}$ & 0 & 0.22 & 0.20 & 0.04 & 0.46 \\
\hline & $3.986^{\mathrm{a}}$ & $2^{+}$ & 2 & 0.67 & 0.52 & 0.10 & 1.30 \\
\hline & $4.088^{\mathrm{a}}$ & $3^{+}$ & 3 & 0.75 & 0.58 & 0.11 & 1.44 \\
\hline & \multirow[t]{2}{*}{$4.142 \%^{\mathrm{a}}$} & $4^{+}$ & 4 & 1.56 & 1.19 & 0.23 & 2.97 \\
\hline & & \multicolumn{3}{|l|}{ Inclusive } & & & 16.46 \\
\hline$\left({ }^{19} \mathrm{C},{ }^{17} \mathrm{C}\right)[13]$ & \multirow[t]{2}{*}{0.000} & \multirow[t]{2}{*}{$3 / 2^{+}$} & 0 & 0.05 & 0.04 & 0.01 & 0.10 \\
\hline \multirow{6}{*}{$S_{1 n}(17)=0.727$} & & & 1 & 0.47 & 0.38 & 0.08 & 0.93 \\
\hline & \multirow[t]{2}{*}{0.032} & \multirow[t]{2}{*}{$5 / 2^{+}$} & 2 & 1.92 & 1.70 & 0.38 & 3.99 \\
\hline & & & 3 & 1.51 & 1.28 & 0.27 & 3.06 \\
\hline & \multirow[t]{3}{*}{0.295} & $1 / 2^{+}$ & 1 & 1.88 & 1.59 & 0.34 & 3.81 \\
\hline & & & 2 & 0.04 & 0.03 & 0.01 & 0.08 \\
\hline & & Inclusive & & & & & 11.97 \\
\hline
\end{tabular}

${ }^{\mathrm{a}}$ Experimental energy used for this state.

not been explored further here and do not affect the conclusions of the present analysis.

These trends across the carbon isotopic chain are summarized in Fig. 4. The dotted and dashed lines connect the calculated $\sigma_{-2 n(d)}$ and $\sigma_{-1 n(e)}$, respectively, with their sum given by the solid line. When including the additional shell model states (within $1.5 \mathrm{MeV}$ above the $A-1$ twoneutron threshold), the cross sections are given by the open inverted triangles. The trends of the data are qualitatively well reproduced, except in the case of ${ }^{15} \mathrm{C}$. The staggering of the theoretical cross section is largely due to the staggering of the total spectroscopic strength for one-neutron removal to unbound states, the direct consequence of the staggered energy windows and one-neutron separation energies. The single-particle cross sections for these reasonably well-bound states exhibit no significant staggering with $A$.

The experimental data are not well reproduced in the case of two-neutron removal from ${ }^{15} \mathrm{C}$. While the theoretical calculation deviates from the experimental trend, it is consistent with that suggested by the size of the final state energy windows, shown in Fig. 2. We note that the one-neutron separation energy for ${ }^{14} \mathrm{C}$ is large, so the states in ${ }^{14} \mathrm{C}$ that contribute to $\sigma_{-1 n(e)}$ are relatively high in excitation when compared with other examples discussed here, i.e., between 


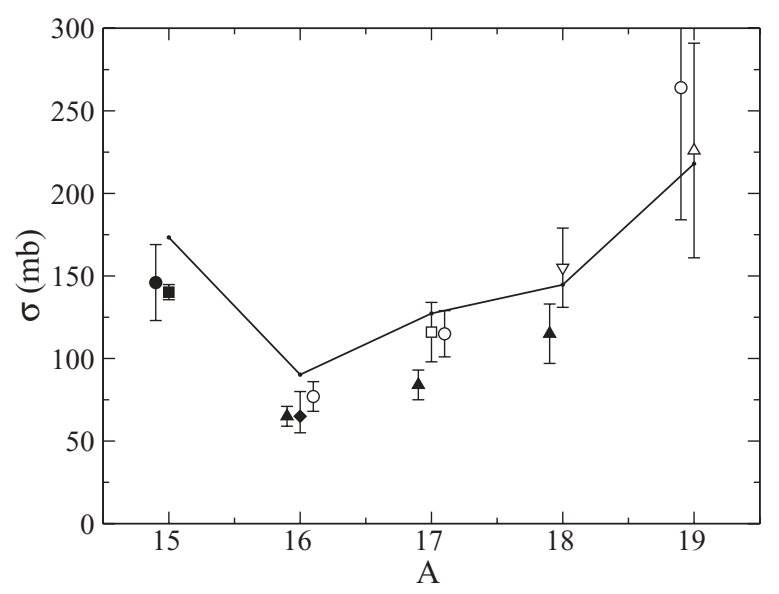

FIG. 3. Calculated and measured inclusive one-neutron removal cross sections for the carbon isotopes. The solid line joins the theoretical calculations of $\sigma_{-1 n}$ for each isotope. The calculations are for a ${ }^{12} \mathrm{C}$ target for the ${ }^{16} \mathrm{C},{ }^{17} \mathrm{C}$, and ${ }^{18} \mathrm{C}$ projectiles at 83,79 , and $80 \mathrm{MeV} /$ nucleon, respectively, and for a ${ }^{9} \mathrm{Be}$ target for ${ }^{15} \mathrm{C}$ and ${ }^{19} \mathrm{C}$ at 103 and $64 \mathrm{MeV} /$ nucleon. The measured cross sections are for ${ }^{15} \mathrm{C}$ [12,34] (solid squares, solid circles), ${ }^{16} \mathrm{C}$ [9-11] (open circles, solid diamonds, solid triangles), ${ }^{17} \mathrm{C}[9,11,14]$ (open circles, solid triangles, open squares), ${ }^{18} \mathrm{C}[11,15]$ (solid triangles, open inverted triangles) and ${ }^{19} \mathrm{C}[9,13]$ (open circles, open triangles).

8.176 and $13.122 \mathrm{MeV}$. Our calculations are consistent with the small spectroscopic strength we would expect to find at these energies. To reproduce the experimental data point would require an increase of the total spectroscopic strength in this energy window by at least a factor of 2 .

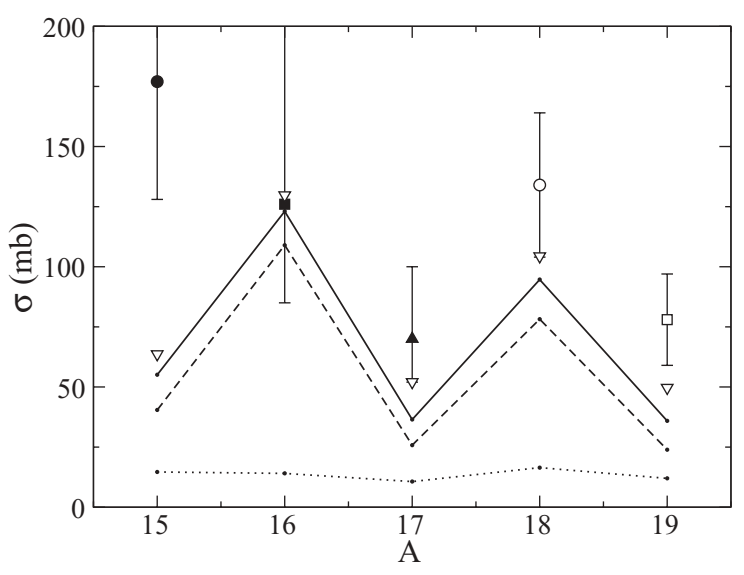

FIG. 4. Calculated and measured inclusive two-neutron removal cross sections for the carbon isotopes. The lines join the results from the direct (dotted line and Table III) and indirect one-neutron knockout to unbound intermediate states (dashed line and Table II), two-neutron removal mechanisms. The solid line shows the summed cross sections. The open inverted triangles show the total cross sections when including the additional contributions from shell model states predicted to lie above but within $1.5 \mathrm{MeV}$ of the two-neutron threshold in the mass $A-1$ system. The calculations are for a ${ }^{12} \mathrm{C}$ target for the ${ }^{15} \mathrm{C},{ }^{16} \mathrm{C},{ }^{17} \mathrm{C}$, and ${ }^{18} \mathrm{C}$ projectiles, at $83,83,79$, and $80 \mathrm{MeV} /$ nucleon, respectively, and for a ${ }^{9} \mathrm{Be}$ target for ${ }^{19} \mathrm{C}$ at 64 $\mathrm{MeV} /$ nucleon. The measured cross sections are for ${ }^{15} \mathrm{C}[34],{ }^{16} \mathrm{C}[10]$, ${ }^{17} \mathrm{C}[14],{ }^{18} \mathrm{C}$ [15], and ${ }^{19} \mathrm{C}$ [13].

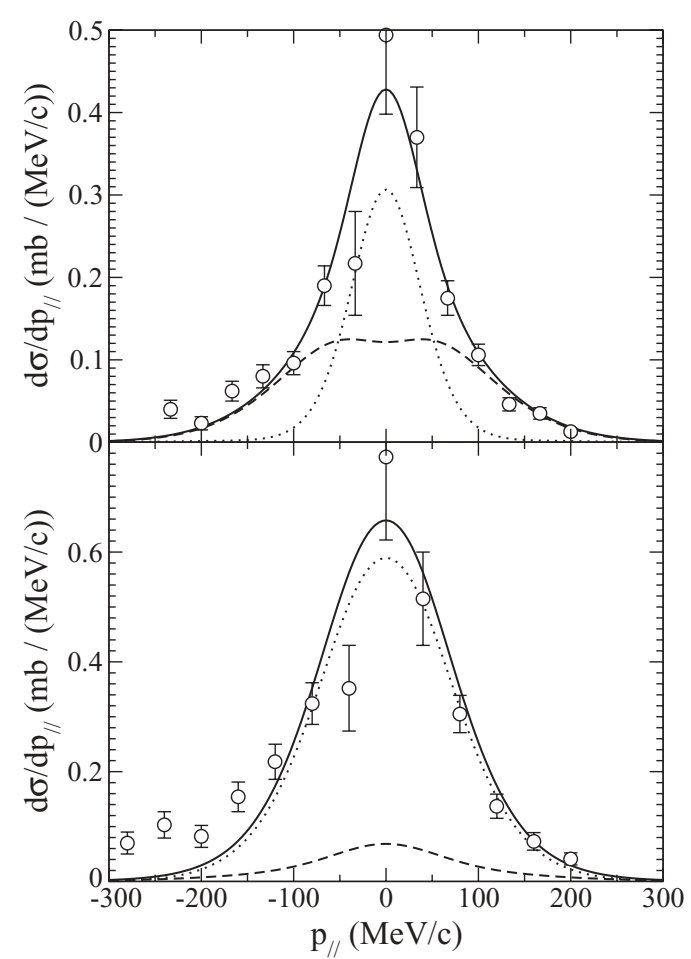

FIG. 5. Theoretical parallel momentum distributions for one- and two-neutron removal from ${ }^{16} \mathrm{C}$, compared with the data of Ref. [10]. The one-neutron distributions (upper panel) are calculated based on the stripping mechanism. Shown are the distributions for the ground state (dotted line, $\ell=0$ ) and $0.740 \mathrm{MeV}$ excited state (dashed line, $\ell=2$ ) transitions. The results shown include the deduced shell model suppression factor $R_{s}$ from Table I. The two-neutron (lower panel) removal distribution (solid line) is the sum of indirect (dotted line) and direct (dashed line) two-neutron knockout contributions. The calculated parallel momentum distributions have not been folded with the experimental resolutions, though the expected broadening is rather small.

\section{Results for parallel momentum distributions}

Parallel momentum distributions for one-nucleon removal have a shape characteristic of the orbital angular momentum $\ell$ of the removed nucleon. The parallel momentum distribution following direct two-nucleon knockout has a width characteristic of the coupling of the two removed nucleons in the initial state $[18,19]$. In calculating the two-nucleon removal parallel momentum distributions, we sum the contributions arising from the direct and indirect processes. For the latter process, we use the initial one-nucleon knockout distribution, assuming this is unaffected by the subsequent (low-energy) evaporation process and that any broadening due to evaporation is small. Uncertainties in the energies of excited states will also affect the widths of the theoretical parallel momentum distributions, final states of higher excitation giving broader momentum distributions.

Figures 5 and 6 show the parallel momentum distributions for one- and two-neutron knockout from ${ }^{16} \mathrm{C}$ and ${ }^{19} \mathrm{C}$, respectively. The one-neutron removal distributions show contributions arising from $\ell=0$ (dotted) and $\ell=2$ (dashed) neutron knockout. These include the shell model suppression 


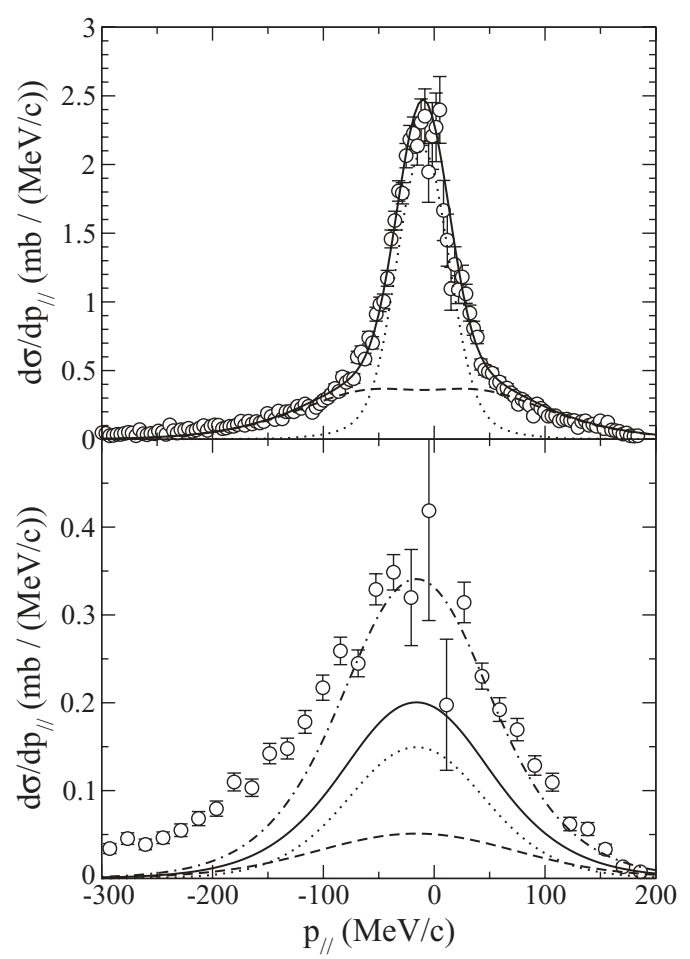

FIG. 6. Theoretical parallel momentum distributions for oneand two-neutron removal from ${ }^{19} \mathrm{C}$, compared with the data of Ref. [13]. The one-neutron distribution (upper panel) is calculated based on the stripping mechanism and is scaled to the measured integrated inclusive cross section. The calculation had to be offset by $-10 \mathrm{MeV} / \mathrm{c}$ to overlay the data. The two-neutron (lower panel) removal cross section (solid line) includes contributions from the indirect (dotted line) and direct (dashed line) mechanisms. The dotted-dashed curve results when the total theoretical distribution is scaled to the data. The two-neutron removal distributions had to be offset by $-16 \mathrm{MeV} / c$.

factor $R_{s}$ derived from the inclusive cross sections. All of the final states shown in Table I were included in the calculations and have been summed according to their orbital angular momentum for simplicity. The relative strengths of $s$ - and $d$-wave components are not fitted and are essentially given by the shell model spectroscopic factors. The strong $s$-wave component in the ${ }^{19} \mathrm{C} \rightarrow{ }^{18} \mathrm{C}$ distribution is consistent with the now accepted $J^{\pi}=1 / 2^{+}$ground-state assignment for ${ }^{19} \mathrm{C}$.

Despite our assumed lack of broadening of the distribution from the evaporation step of the indirect two-neutron removal mechanism, the experimental distributions for ${ }^{16} \mathrm{C} \rightarrow{ }^{14} \mathrm{C}$ and ${ }^{19} \mathrm{C} \rightarrow{ }^{17} \mathrm{C}$ are qualitatively well described. In both cases, the largest contributions arise from $\ell=1$ indirect knockout (see Table II). The ${ }^{16} \mathrm{C} \rightarrow{ }^{14} \mathrm{C}$ distribution shows surprisingly good quantitative agreement, and while the ${ }^{19} \mathrm{C} \rightarrow{ }^{17} \mathrm{C}$ case shows a clear underestimate in the absolute magnitude of the cross section, scaling the calculated distribution shows good qualitative agreement with the measured shape. The low-momentum tails visible in the experimental data are not reproduced; the sudden eikonal model is explicitly energy nonconserving and always produces distributions symmetric about zero momentum in the projectile rest frame.

\section{SUMMARY AND CONCLUSIONS}

In summary, one- and two-neutron removal cross sections from the neutron-rich carbon isotopes have been calculated using the eikonal reaction plus shell model structure models. The one-neutron removal cross sections are in good agreement with the published experimental values. The two-neutron removal cross sections were calculated by considering the removal of one-neutron to an unbound state in the $A-1$ daughter, with the assumption that this unbound state will decay by neutron emission to bound states in the $A-2$ residue. Fully correlated direct two-neutron removal cross section were also calculated, and it was shown that the two-neutron removal process is dominated by the former one-neutron removal to unbound states mechanism. The staggering in the experimental two-neutron removal cross sections, understood as arising from the staggering of the neutron separation energies, is reproduced qualitatively. The published ${ }^{15} \mathrm{C} \rightarrow{ }^{13} \mathrm{C}$ data point does not follow the trend suggested by these general arguments or the systematics of the other odd- $A$ isotopes. More experimental information on this reaction, with higher statistics, would thus be of value. The available inclusive parallel momentum distributions of the reaction residues following one- and two-neutron knockout from ${ }^{16} \mathrm{C}$ and ${ }^{19} \mathrm{C}$ are also well reproduced by the theoretical calculations.

Our calculations for two-neutron removal are in general smaller than experimental observations, particularly when considering the expected (modest) suppression of the shell model strengths used. However, the error bars on the twoneutron removal cross sections are also appreciable. We note that we have used only an estimate of the direct, two-neutron elastic breakup contributions, as used previously elsewhere. This suggests that this mechanism enters at the $10 \%$ level to the (already small) direct two-neutron removal component. An improved estimate would nevertheless be valuable in the future, in particular, if and when exclusive measurements that allow one to isolate the direct contribution become feasible. We have shown clearly and quantitatively that the single-neutron knockout plus evaporation mechanism dominates over the direct two-nucleon removal mechanism in situations where the removed nucleons are initially weakly bound. This makes investigations of two-neutron correlations in such systems using this technique much more difficult. Such two-step processes will also be important in reactions that remove unlike nucleons, $n p$ pairs, from exotic nuclei, where one of the nucleons must necessarily lie near a weakly bound Fermi surface.

\section{ACKNOWLEDGMENTS}

We thank Professor B. Alex Brown for his continued advice upon the shell model calculations used and Professor Akira Ozawa for valuable discussions and for providing the new data point for ${ }^{18} \mathrm{C}$ in advance of its publication. This work was supported by the UK Science and Technology Facilities Council (STFC) under Grants EP/D003628 and ST/F012012. 
[1] J. A. Tostevin, J. Phys. G 25, 735 (1999).

[2] P. G. Hansen and J. A. Tostevin, Annu. Rev. Nucl. Part. Sci. 53, 219 (2003).

[3] A. Gade, P. Adrich, D. Bazin, M. D. Bowen, B. A. Brown, C. M. Campbell, J. M. Cook, T. Glasmacher, P. G. Hansen, K. Hosier et al., Phys. Rev. C 77, 044306 (2008).

[4] D. Bazin, B. A. Brown, C. M. Campbell, J. A. Church, D. C. Dinca, J. Enders, A. Gade, T. Glasmacher, P. G. Hansen, W. F. Mueller et al., Phys. Rev. Lett. 91, 012501 (2003).

[5] J. A. Tostevin, G. Podolyák, B. A. Brown, and P. G. Hansen, Phys. Rev. C 70, 064602 (2004).

[6] K. Yoneda, A. Obertelli, A. Gade, D. Bazin, B. A. Brown, C. M. Campbell, J. M. Cook, P. D. Cottle, A. D. Davies, D.-C. Dinca et al., Phys. Rev. C 74, 021303(R) (2006).

[7] J. A. Tostevin and B. A. Brown, Phys. Rev. C 74, 064604 (2006).

[8] D. Bazin, W. Benenson, B. A. Brown, J. Brown, B. Davids, M. Frauerbach, P. G. Hansen, P. Mantics, D. J. Morrissey, C. F. Powell et al., Phys. Rev. C 57, 2156 (1998).

[9] V. Maddalena, T. Aumann, D. Bazin, B. A. Brown, J. A. Caggiano, B. Davids, T. Glasmacher, P. Hansen, R. W. Ibbotson, A. Navin et al., Phys. Rev. C 63, 024613 (2001).

[10] T. Yamaguchi, T. Zheng, A. Ozawa, M. Chiba, R. Kanungo, T. Kato, K. Morimoto, T. Ohnishi, T. Suda, Y. Yamaguchi et al., Nucl. Phys. A724, 3 (2003).

[11] E. Sauvan, F. Carstoiu, N. A. Orr, J. S. Winfield, M. Freer, J. C. Angélique, W. N. Catford, N. M. Clarke, N. Curtis, S. Grévy et al., Phys. Rev. C 69, 044603 (2004).

[12] J. R. Terry, D. Bazin, B. A. Brown, J. Enders, T. Glasmacher, P. G. Hansen, B. M. Sherrill, and J. A. Tostevin, Phys. Rev. C 69, 054306 (2004).

[13] M. Chiba, R. Kanundo, B. Abu-Ibrahim, S. Adhikari, Q. Q. Fang, N. Iwasa, K. Kimura, K. Maeda, S. Nishimura, T. Ohnishi et al., Nucl. Phys. A741, 29 (2004).

[14] C. Wu, Y. Yamaguchi, A. Ozawa, I. Tanihata, D. Jiang, H. Hua, T. Zheng, Z. Li, and T. Ye, J. Phys. G 31, 39 (2005).

[15] A. Ozawa, D. Q. Fang, M. Fukuda, N. Iwasa, T. Izumikawa, H. Jeppesen, R. Kanungo, R. Koyama, T. Ohnishi, T. Ohtsubo et al., Phys. Rev. C 78, 054313 (2008).

[16] J. A. Tostevin, Nucl. Phys. A682, 320c (2001).

[17] G. Audi, A. H. Wapstra, and C. Thibault, Nucl. Phys. A729, 337 (2003).

[18] J. A. Tostevin, Eur. Phys. J. Spec. Top. 150, 67 (2007).
[19] E. C. Simpson and J. A. Tostevin (in preparation).

[20] H. Esbensen and G. F. Bertsch, Phys. Rev. C 64, 014608 (2001); G. F. Bertsch and H. Esbensen, Prog. Theor. Phys. Suppl. 146, 319 (2002).

[21] J. M. Brooke, J. S. Al-Khalili, and J. A. Tostevin, Phys. Rev. C 59, 1560 (1999).

[22] J. A. Tostevin, J. M. Brooke, J. Mortimer, and I. J. Thompson, Prog. Theor. Phys. Suppl. 146, 338 (2002).

[23] J. S. Al-Khalili, J. A. Tostevin, and I. J. Thompson, Phys. Rev. C 54, 1843 (1996).

[24] B. A. Brown, Phys. Rev. C 58, 220 (1998).

[25] L. Ray, Phys. Rev. C 20, 1857 (1979).

[26] J. A. Tostevin, in Fission and Properties of Neutron-Rich Nuclei, Proceedings of the Second International Conference, St. Andrews, Scotland, 28 June-3 July 1999, edited by J. H. Hamilton, W. R. Phillips, and H. K. Carter (World Scientific, Singapore, 2000), p. 429.

[27] J.-P. Jeukenne, A. Lejeune, and C. Mahaux, Phys. Rev. C 16, 80 (1977).

[28] D. Bazin et al., in Proceedings of the International Nuclear Physics Conference (INPC07), Tokyo, 2007, Vol. 2, p. 406 (unpublished); D. Bazin et al. (submitted to Phys. Rev. Lett.).

[29] J. Enders, T. Baumann, B. A. Brown, N. H. Frank, P. G. Hansen, P. R. Heckman, B. M. Sherrill, A. Stolz, M. Thoennessen, J. A. Tostevin, E. J. Tryggestad, S. Typel, and M. S. Wallace, Phys. Rev. C 67, 064301 (2003).

[30] B. A. Brown, A. Etchegoyen, N. S. Godwin, W. D. Rae, W. Richter, W. E. Ormand, E. K. Warburton, J. S. Winfield, L. Zhao, and C. H. Zimmerman, OXBASH for Windows (2004), MSU-NSCL Rep. No. 1289 (unpublished).

[31] E. K. Warburton and B. A. Brown, Phys. Rev. C 46, 923 (1992).

[32] A. E. Dieperink and T. de Forest Jr., Phys. Rev. C 10, 543 (1974).

[33] M. Stanoiu, D. Sohler, O. Sorlin, F. Azaiez, Z. Dombrádi, B. A. Brown, M. Belleguic, C. Borcea, C. Bourgeois, Z. Dlouhy et al., Phys. Rev. C 78, 034315 (2008).

[34] D. Q. Fang, T. Yamaguchi, T. Zheng, A. Ozawa, M. Chiba, R. Kanungo, T. Kato, K. Morimoto, T. Ohnishi, T. Suda et al., Phys. Rev. C 69, 034613 (2004).

[35] A. Ozawa, T. Suzuki, and I. Tanihata, Nucl. Phys. A693, 32 (2001). 